\title{
稀土离子促进胰岛素经大鼠肺部吸收及 降血糖的效应
}

\section{程 驿 沈赞聪 张强 魏树礼 李荣昌 王 滳*}

(北京医科大学药学院, 北京 100083 .* 联系人)

摘要 为了探讨稀土作为胰岛素吸收促进剂的可能性, 以先给稀土后给胰岛素和混合给药两 种方法研究了稀土离子对胰岛素经大鼠肺部吸收及降血糖效应的促进作用。实验结果表明, 与单独给胰岛素相比, 稀土阳离子能有效地提高血液中胰岛素的水平, 增加血糖下降幅度. 促 进胰岛素吸收和降低血糖的程度与稀土的物种和浓度有关. 并对其作用机理进行了讨论.

\section{关键词 稀土 胰岛素 血糖 大鼠}

稀土的一个重要细胞生物效应是能在极低浓度下增强细胞的通透性 ${ }^{[1,2]}$. 这一性质有可 能用于增加细胞对药物分子的摄入. Canada 等人 ${ }^{[3]}$ 报道用 $\mathrm{Tb}^{3+}$ 能促进顺铂在人卵巢癌细胞 中的积累, 进而增强它的药效. 但更有意义的是利用它增加大分子如肽类的生物利用度. 已 有报道指出, 氯化钎 (浓度为 $10^{-5} \mathrm{~mol} / \mathrm{L}$ ) 能增加质粒( PBR322) 在 $E . c o l i$ 中的转化 ${ }^{[4]}$. 显然稀 土在适当条件下可以促进生物大分子的跨膜转运, 但是如何控制条件使其有利于给定大分子 的摄入还需进一步研究. 最近我们发现, 稀土-红细胞相互作用产生域结构和打洞, 并由此导 致通透性不同程度的增加. 这一过程是依赖浓度的, 而且是三时相的过程. 在浓度适当时可 以使蛋白质通过细胞膜, 而细胞完整性不变，并且除去表面结合的稀土又可重封膜上的 洞 $^{[2,5 \sim 8]}$. 因此, 可以设想利用稀土离子调控细胞膜通透性的方法来增加肽类药物的吸收和 向靶细胞内运输, 从而提高细胞内药物的浓度. 这对药物的有效吸收和利用具有重要的意义.

如何有效地提高非注射给药途径的胰岛素在体内的吸收和利用一直是一个被普遍关注的 问题. 口服给胰岛素易被酶降解, 吸收很少. 与之相比, 通过肺部给胰岛素具有吸收面积大, 肺泡上皮细胞膜屏障较薄, 胰岛素降解酶较少等优点 ${ }^{[9 \sim 11]}$. 但是与注射相比, 其生物利用度 仍然较低. 本文采用 Wistar 大鼠肺部给药为模型, 研究了稀土离子对胰岛素经肺吸收的促进 作用和降血糖效应.

\section{1 材料与方法}

纯种 Wistar 雄性大鼠 (北京医科大学实验动物部提供), 氯化稀土( $\mathrm{LnCl}_{3}$ ) 和柠檬酸稀土配 合物( [ $\left.\mathrm{Ln}(\text { Citrate })_{2}\right]^{3-}$ ) 溶液由相应的 $\mathrm{Ln}_{2} \mathrm{O}_{3}(99.99 \%)$ 制得. 制备和浓度标定方法与文献 $[5,6]$ 相同. 含锌猪胰岛素 $(26 \mathrm{IU} / \mathrm{mg})$ 购自徐州生化试剂厂; ${ }^{125} \mathrm{~F}$ 胰岛素放免试剂盒购自北京 原子能研究院. 葡萄糖氧化酶混合试剂为 $100 \mathrm{U} / \mathrm{mg}$ 葡萄糖氧化酶, $1.5 \mathrm{mg}$ 过氧化物酶, $25 \mathrm{mg}$ 4-氨基安替比林和 $250 \mathrm{mg}$ 叠氮化钠在 $250 \mathrm{~mL} \mathrm{pH}=7.0$ 的 Tris $\mathrm{HCl}(50 \mathrm{mmol} / \mathrm{L})$ 中的溶液. 其他 试剂均为分析纯. 实验所用溶液均用灭菌去离子双蒸水配制.

Wistar 雄性大鼠( 体重为 250 $450 \mathrm{~g}$ ) 实验前禁食 12 $16 \mathrm{~h}$. 用 $2 \%$ 戊巴比妥钠 $(40 \mathrm{mg} / \mathrm{kg}$ ) 麻醉后, 将大鼠仰卧固定, 手术切开气管. 按每 $400 \mathrm{~g}$ 体重 $25 \mu \mathrm{L}$ 的剂量自甲状软骨下第 5 至 
第 6 管环之间注入一定浓度的稀土溶液 ${ }^{[10]}, 30 \mathrm{~min}$ 后, 再按每 $400 \mathrm{~g}$ 体重 $40 \mu \mathrm{L}$ 的剂量向肺内 注入 $10 \mathrm{IU} / \mathrm{mL}$ 肤岛素溶液. 或者以同样方式注入胰岛素和稀土离子的混合液. 相应的对照 大鼠注入含相同浓度稀土离子的等体积生理盐水．在不同的时间间隔内分别从大鼠的尾尖取 $0.5 \mathrm{~mL}$ 血.

血样经凝固后以 $3000 \mathrm{r} / \mathrm{min}$ 离心 $10 \mathrm{~min}$ ，立即吸取 $20 \mu \mathrm{L}$ 血清用文献 [ 10] 所描述的葡萄 糖氧化酶珐测定血糖值. 剩余的血清于- $20^{\circ} \mathrm{C}$ 冷冻保存, 在 $2 \mathrm{~d}$ 内按文献[ 11] 所述的放射免 疫法测定其胰岛素水平. 胰岛素的生物利用度和血糖药理生物利用度的计算采用梯形法 ${ }^{[12]}$ :

胰岛素生物利用度 $(F)=\left(A U C_{\mathrm{it}} / \mathrm{dos}_{\mathrm{it}}\right) /\left(A U C_{\mathrm{sc}} /\right.$ dose $\left._{\mathrm{sc}}\right)$

血糖药理生物利用度 $(\mathrm{PA} \%)=\left(A A C_{\mathrm{it}} / \mathrm{dose}_{\mathrm{it}}\right) /\left(A A C_{\mathrm{sc}} / \mathrm{dose}_{\mathrm{sc}}\right)$

式中 $A U C$ 和 $A A C$ 表示胰岛素浓度-时间曲线下的面积和血糖降低-时间曲线上的面积, it 和 sc 分别表示肺部给药和皮下给药方式. dose 表示给胰岛素的剂量. 所得实验数据均为 5 只大鼠 的平均值. 误差分析采用 Student's $t$-检验法, $P<0.05$.

\section{2 结果}

为了研究稀土离子对胰岛素经肺部吸收和其后续降血糖作用的影响, 我们选择了 4 种稀 土, 考察了稀土离子在不同存在形式和不同给药方式下的效应. 与此平行, 进行只给稀土的对 照实验, 以排除稀土对体内胰岛素的分泌和血糖水平的影响.

2.1 稀土离子对胰岛素经肺吸收的促进作用

图 1 表示 $\mathrm{Gd}^{3+}$ 和 $\left[\mathrm{Gd} \text { (Citrate) }{ }_{2}\right]^{3-}$ 对胰岛素经肺部吸收随时间变化的影响. 从中可见, 单 独给胰岛素的情况下, 血清中胰岛素含量在 $0.5 \mathrm{~h}$ 后达到最大值, 随后逐渐降低. 若先给 (0.04 $0.6 \mathrm{mg} / \mathrm{kg}$ 体重) 的 $\mathrm{Gd}^{3+}, 30 \mathrm{~min}$ 后再给相同浓度的胰岛素, 则血清中胰岛素含量所达 到的最大值高于前者, 而且胰岛素随时间降低的速度也明显减缓(图 1(a))。在相同浓度和条 件下, $\left[\mathrm{Gd} \text { (Citrate) }{ }_{2}\right]^{3-}$ 组胰岛素含量所达到的最大值高于 $\mathrm{Gd}^{3+}$ 组. 若把 $\mathrm{Gd}^{3+}$ 和胰岛素事先
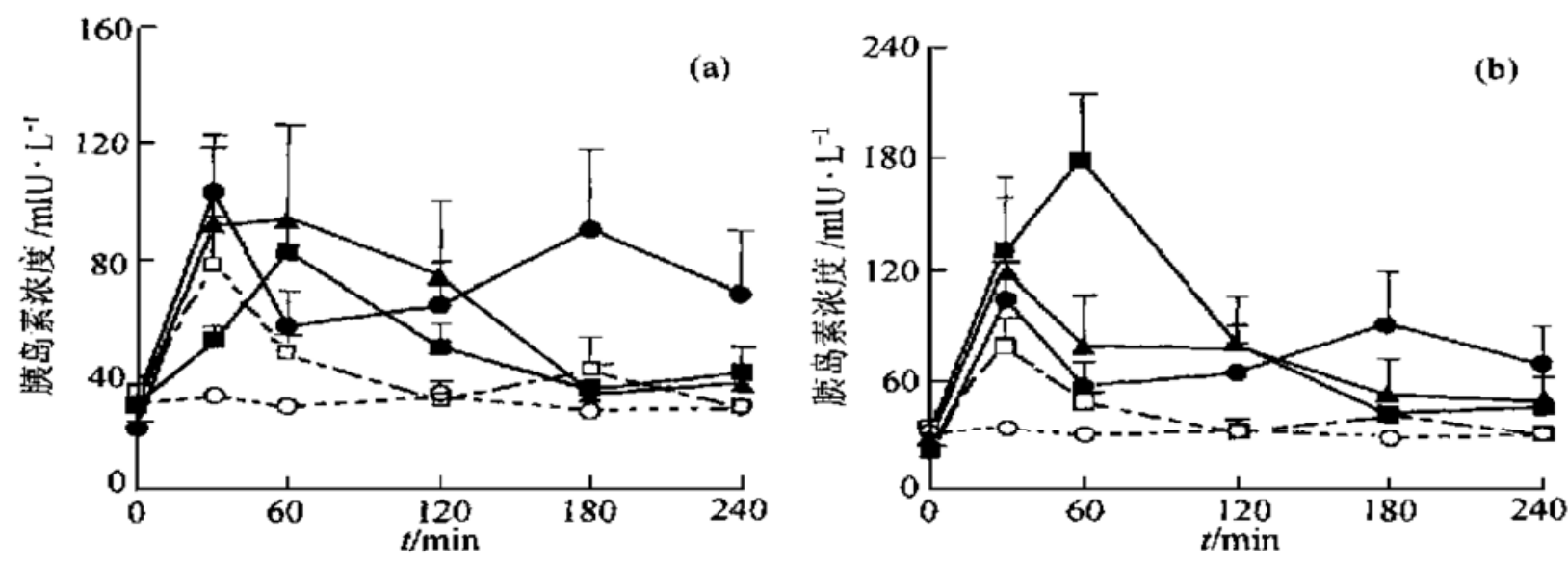

图 $1 \mathrm{Gd}$ 物种对经肺部吸收胰岛素后血清胰岛素水平的影响 $\mathrm{O—}$ —单独给 $\mathrm{Gd}^{3+}, \square$ —单独给胰岛素 $(\mathrm{pH}=7.0)$; (a) 先给 $\mathrm{Gd}^{3+} 30 \mathrm{~min}$ 后, 再给胰岛素 $\left(\boldsymbol{a}-40 \mathrm{\mu}_{\mathrm{g}} / \mathrm{kg} \mathrm{Gd} \mathrm{gd}^{3+}\right.$, $\left.-0.2 \mathrm{mg} / \mathrm{kg} \mathrm{Gd}^{3+}, \boldsymbol{\Delta} \longrightarrow 0.6 \mathrm{mg} / \mathrm{kg} \mathrm{Gd}^{3+}\right)$; (b) 先给 $\mathrm{Gd}$ 物种 $30 \mathrm{~min}$ 后, 再给胰岛素 $\left(\bullet-0.2 \mathrm{mg} / \mathrm{kg} \mathrm{Gd} \mathrm{G}^{3+}\right.$, $\left.\boldsymbol{\Delta} \longrightarrow 0.2 \mathrm{mg} / \mathrm{kg}\left[\mathrm{Gd}(\text { citrate })_{2}\right]^{3-}\right), \boldsymbol{\square} \longrightarrow 0.2 \mathrm{mg} / \mathrm{kg} \mathrm{Gd} \mathrm{d}^{3+}$ 和胰岛素 $(\mathrm{pH}=7.0)$ 混合给药( 胰岛素剂量均为 1.0 $\mathrm{IU} / \mathrm{kg}, n=5$, 实验误差: SE, $P<0.05$ 或 0.01 ) 
混合后再给药, 则血清中胰岛素含量所达最大值的时间滞后到 $1 \mathrm{~h}$ 且最大值远高于分开给 $\mathrm{Gd}^{3+}$ (或 $\left[\mathrm{Gd}(\text { Citrate })_{2}\right]^{3-}$ ) 和胰岛素组( 图 1(b) ). 稀土离子对胰岛素经肺部吸收的促进作用 从胰岛素生物利用度中清晰可见 (表 1)。在相同浓度下, 稀土与胰岛素混合后给药的促进作 用最大. 对于不同稀土, $\mathrm{Gd}^{3+}>\mathrm{Ce}^{3+}>\mathrm{La}^{3+}>\mathrm{Lu}^{3+}$. 对同一稀土离子 $\left(\mathrm{Gd}^{3+}\right)$ 来说, 促进胰岛 素吸收的作用随稀土浓度增加而增加, 达最大值后又下降。对照实验结果表明, 在实验所用的 稀土浓度范围内, 仅给稀土组在 $4 \mathrm{~h}$ 内大鼠血清中胰岛素和血糖的水平未变.

表 1 稀土离子对胰岛素生物利用度和血糖药理利用度的影响a)

\begin{tabular}{|c|c|c|c|c|c|c|}
\hline & \multicolumn{3}{|c|}{ 胰岛素 } & \multicolumn{3}{|c|}{ 血糖 } \\
\hline$A U C$ & $\mathrm{IU} \cdot \mathrm{L}^{-1} \cdot \min ^{-1}$ & $\pm \mathrm{SE}$ & $F / \%$ & $A A C / \% \cdot \mathrm{h}$ & $\pm \mathrm{SE}$ & $\mathrm{PA} / \%$ \\
\hline $1.0 \mathrm{IU} / \mathrm{kg}$ 胰岛素 sc & 17229.6 & 1598.1 & 100 & 363.3 & 0.0 & 100 \\
\hline $1.0 \mathrm{IU} / \mathrm{kg}$ 胰岛素 it & 2323.2 & 502.9 & 13.4 & 84.5 & 15.4 & 23.3 \\
\hline$\#+0.2 \mathrm{mg} / \mathrm{kg} \mathrm{La}^{3+}$ & 5347.2 & 645.1 & 30.9 & 129.0 & 22.2 & 35.5 \\
\hline$\#+0.2 \mathrm{mg} / \mathrm{kg} \mathrm{Ce}^{3+}$ & 10012.2 & 968.0 & 57.9 & 104.4 & 19.8 & 28.7 \\
\hline$\#+0.2 \mathrm{mg} / \mathrm{kg} \mathrm{Lu}^{3+}$ & 2709.0 & 456.5 & 15.6 & 91.2 & 17.7 & 25.1 \\
\hline$\#+40 \mu \mathrm{g} / \mathrm{kg} \mathrm{Gd}^{3+}$ & 6817.8 & 1352.0 & 39.4 & 117.0 & 4.3 & 32.2 \\
\hline$\#+0.2 \mathrm{mg} / \mathrm{kg} \mathrm{Gd}^{3+}$ & 2709.0 & 456.5 & 59.5 & 131.8 & 16.5 & 36.3 \\
\hline$\#+0.6 \mathrm{mg} / \mathrm{kg} \mathrm{Gd}^{3+}$ & 8016.6 & 1692.4 & 46.3 & 130.1 & 32.9 & 35.8 \\
\hline$\#+0.2 \mathrm{mg} / \mathrm{kg}\left[\mathrm{Gd}(\mathrm{cit})_{2}\right]^{3-}$ & 11836.8 & 2221.1 & 68.4 & 82.7 & 19.1 & 22.7 \\
\hline $\begin{array}{l}1.0 \mathrm{IU} / \mathrm{kg} \text { 胰岛素和 } \mathrm{Gd}^{3+} \\
(0.2 \mathrm{mg} / \mathrm{kg}) \text { 混合给药 } \\
\end{array}$ & 13860.0 & 1264.0 & 80.1 & 94.72 & 19.0 & 26.1 \\
\hline
\end{tabular}

a)\# 表示先肺部给稀土 $30 \mathrm{~min}$ 后, 再给 $1.0 \mathrm{IU} / \mathrm{kg}$ 肤岛素, $n=5$

2.2 稀土离子对血糖水平的影响

图 2 表示稀土对血糖含量随时间变化的影响. 只给稀土, 既不影响胰岛素水平, 在 $4 \mathrm{~h}$ 对
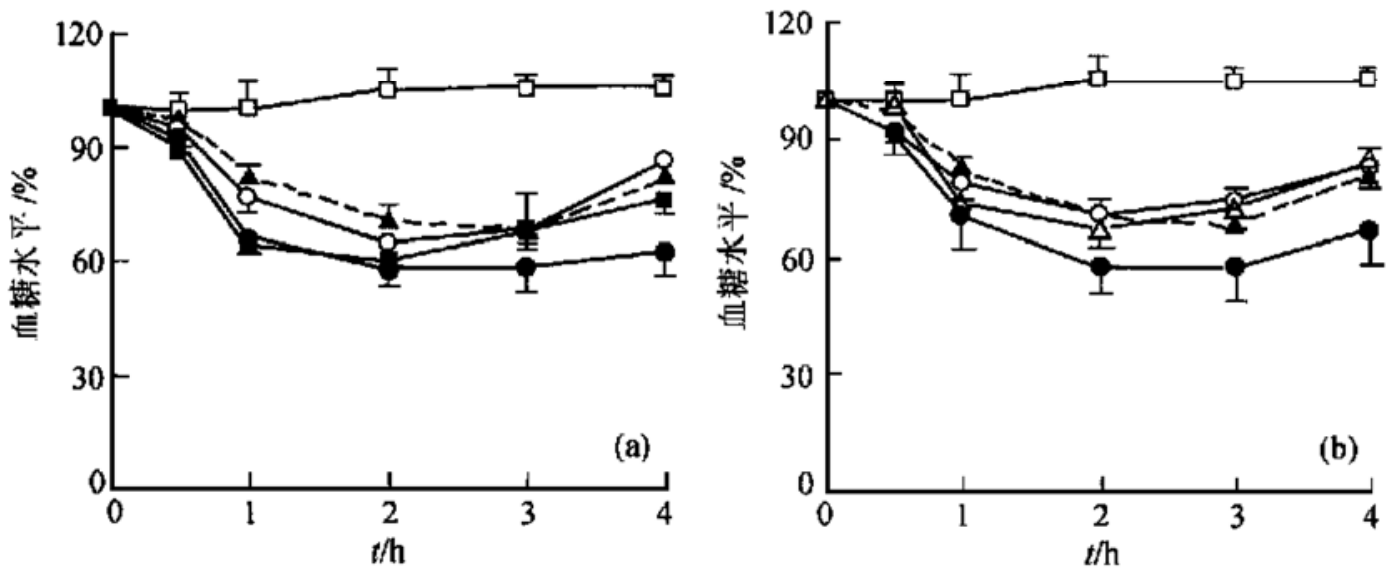

图 2 稀土物种在促进胰岛素吸收过程中对血糖的影响

$\square$ —单独给 $\mathrm{Ln}^{3+}, \boldsymbol{\Delta}$ —单独给胰岛素 $(\mathrm{pH} 7.0)$; (a) $\mathrm{O}-0.2 \mathrm{mg} / \mathrm{kg} \mathrm{Gd}$ 和胰岛素 $(\mathrm{pH}=$ 7.0) 混合给药, 和 - 分别为 先给 $40 \mu \mathrm{g} / \mathrm{kg}$ 和 $0.2 \mathrm{mg} / \mathrm{kg} \mathrm{Gd}^{3+} 30 \mathrm{~min}$ 后, 再给胰岛素; (b) 先给稀 土物种 $30 \mathrm{~min}$ 后, 再给胰岛素 $\left(\mathrm{O}-0.2 \mathrm{mg} / \mathrm{kg}\left[\mathrm{Gd}\left(\text { citrate) }{ }_{2}\right]^{3-}, \bullet-0.2 \mathrm{mg} / \mathrm{kg} \mathrm{La}{ }^{3+}, \Delta-\right.\right.$

$0.2 \mathrm{mg} / \mathrm{kg} \mathrm{Lu}^{3+}$ ). 胰岛素剂量均为 $1.0 \mathrm{IU} / \mathrm{kg}, n=5$, 实验误差: $\mathrm{SE}, P<0.05$ 或 0.01 
血糖含量也基本上无影响. 单独给胰岛素时, 大鼠血糖含量在 $2 \mathrm{~h}$ 内降到最低水平, 随后逐渐 回升. 给 $\mathrm{Gd}^{3+} 30 \mathrm{~min}$ 后再给胰岛素时, 在 $2 \mathrm{~h}$ 内血糖下降速度明显增快, 随后较高浓度 $(0.2$ $\mathrm{mg} / \mathrm{kg}$ 剂量) 的回升速度显著减缓( 图 $2(\mathrm{a})$ ). 但值得注意的是, 若以 $\mathrm{Gd}^{3+}$ 和胰岛素混合给药, 血糖降低速度虽也提高但却小于先给 $\mathrm{Gd}^{3+}$ 组. 从表 1 中血糖药理生物利用度可见, 先给 0.2 $\mathrm{mg} / \mathrm{kg}$ 剂量 $\mathrm{Gd}^{3+}$ 的降血糖效果比仅给胰岛素组提高了约 $55.92 \%$. 不同稀土离子的降血糖效 率为: $\mathrm{Gd}^{3+}>\mathrm{La}^{3+}>\mathrm{Ce}^{3+}>\mathrm{Lu}^{3+}$. 与稀土氯化物不同, [ Gd(Citrate) $)_{2}^{3-}$ ( $0.2 \mathrm{mg} / \mathrm{kg}$ 剂量) 基本 上无促进经肺给胰岛素的降血糖效果(图 2(b)，表 1).

\section{3 讨论}

胰岛素经内化作用进入细胞. 其经大鼠肺部吸收的主要屏障是肺泡上皮细胞膜. 进入血 液后, 胰岛素被运输到各器官并与相关细胞膜上的胰岛素受体结合, 进而达到降血糖效应 ${ }^{[13]}$, 同时引发一系列生理效应. 实验结果揭示经肺在给胰岛素之前给氯化稀土, 血液中胰岛素生 物利用度增加, 降低血糖水平能力增强. 由于单独给稀土离子在 $4 \mathrm{~h}$ 内并不能导致血清中胰 岛素水平的升高和血糖含量的降低, 因此说明本实验结果是由于稀土离子促进了外源性的胰 岛素经大鼠肺部的吸收. 曾有文献报道 ${ }^{[14]}$, 对患糖尿病的实验大鼠给予低剂量 $(0.05 \mathrm{mg} / \mathrm{kg}$ 体重) $\mathrm{SmCl}_{3} 1.5$ 个月后可明显提高血清中胰岛素的水平和降低血糖. 显然长期连续给低剂量 稀土导致内源性胰岛素的升高与短时间内稀土影响外源性胰岛素的吸收无论在机制上和在效 果上都是不同的. 已有报道, 稀土阳离子能增强细胞通透性 ${ }^{[2 ~ 8]}$. 其机理可能是由于稀土离 子与细胞膜(包括膜蛋白和膜脂) 结合导致膜蛋白构象变化和聚集以及膜脂多相共存, 从而形 成域结构和孔洞结构 ${ }^{[2,5 ~ 8]}$. 稀土离子浓度低时形成域结构, 而浓度高时可以打洞. 这两种 结构与细胞对小或大分子的通透性密切相关 ${ }^{[2,8]}$. 因此推测, 适当浓度的稀土离子之所以能 增加经肺给胰岛素的生物利用度和降低血糖能力可能与它们和肺泡上皮细胞膜结合增加其通 透性, 促进胰岛素的跨膜吸收有关. 这也得到我们体外细胞试验的进一步支持, 我们发现稀土 离子 $\left(\mathrm{Gd}^{3+}\right)$ 可有效地促进胰岛素跨蛙肺泡细胞吸收 (未发表结果). 因为稀土与胰岛素混合 后给药时胰岛素生物利用度更高, 可以认为稀土离子与胰岛素结合后其生成的稀土-胰岛素络 合物更易跨膜吸收. 但其机理有待进一步阐明.

从血糖水平来看, 实验结果 (表 1) 表明, 在给胰岛素前先给氯化稀土都能明显增加胰岛素 的降糖作用. 而且不同正三价稀土离子增强胰岛素吸收效应与其降血糖效应基本上相对应. 不同稀土离子的增强能力不一样, 在所研究的稀土中, $\mathrm{Gd}^{3+}$ 的降血糖效应明显高于其他 $\mathrm{La}^{3+}$, $\mathrm{Ce}^{3+}$ 和 $\mathrm{Lu}^{3+}$. 与此一致, 在增强红细胞通透性方面也是 $\mathrm{Gd}^{3+}$ 的作用最强 ${ }^{[21}$. 但值得注意的 是, [Gd(Citrate) 2$]^{3-}$ 能显著促进胰岛素进入血液, 但并没有导致血糖水平的进一步降低. 这 说明稀土虽均能促进胰岛素的吸收, 导致血液中胰岛素水平升高, 但其降血糖效应与稀土离子 的物种存在形式密切相关. 与上述不同, 当以 $\mathrm{Gd}^{3+}-$ 胰岛素络合物形式给药时虽能有效地促 进胰岛素的吸收, 但血糖水平经下降后回升较快较大. 一方面, 这与它使胰岛素浓度上升很 高后又迅速下降有关(见图 1( (b) ). 另一方面, 可能与 Gd 胰岛素络合物不易与其受体结合 有关. 从图 2(a) 可见, 在混合给药时, 尽管在 $1 \mathrm{~h}$ 时血中胰岛素水平大大超过其他, 但它的降 血糖效果却不如其他.

Williams 等人 ${ }^{15]}$ 曾报道 $\mathrm{Tb}^{3+}$ 能增强胰岛素与细胞膜上的胰岛素受体结合. 稀土降血糖 
效果的大小可以促进胰岛素-受体亲和力为基础来解释. 显然 $\mathrm{Tb}^{3+}$ 既可能与胰岛素结合也可 以与其受体结合. 两者都会或多或少改变胰岛素和受体的构象从而影响两者的结合. 先给稀 土时主要与血液中红细胞膜上的胰岛素受体结合(因另有实验表明, $0.2 \mathrm{mg} / \mathrm{kg}$ 体重的 $\mathrm{Gd}^{3+}$ 经 大鼠肺部可在 $30 \mathrm{~min}$ 后其血液中的 $\mathrm{Gd}$ 含量达到最大值(约 $9 \mathrm{ng} / \mathrm{mL}$ 血清) ), 混合给药时稀土 主要与胰岛素结合, 因此两种给药方式效果不同是可以理解的. 此外, 不能排除稀土物种及其 胰岛素-稀土络合物对血糖代谢过程的不同影响的可能. 有关这些方面的研究正在进行中.

总之, 本文的结果表明, 稀土阳离子能有效提高胰岛素经大鼠肺部吸收的生物利用度, 并 增加其降血糖效应. 稀土阳离子增加细胞膜通透性可能是造成这一生物效应的原因之一, 但 也可能与影响糖代谢有关.

致谢 本工作为国家自然科学基金( 批准号: 29890280) 和国家博士后基金资助项目.

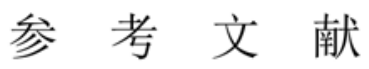

1 王 咅, 韩万书. 中国生物无机化学十年进展. 北京: 高等教育出版社, 1997. 19 31

2 Wang K, Li R C, Cheng Y, et al. Lanthanide the future drugs? Coord Chem Rev, 1999, 190- 192: 297 308

3 Canada R G, Andrews P A, Mack K M, et al. The effects of terbium on the accumulation of cisplatin in human ovarian cancer cells. Biochim Biophys Acta, 1995, 1267: 25 30

4 黄德盈, 吴士筠, 何冬兰, 等. 氯化钎对质粒 PBR322 转化的影响. 第八次全国生物化学和分子生物学学术会议专题报 告汇编. 北京: 北京医科大学出版社, 1998. 231 232

5 Cheng Y, Chen B W, Lu J F, et al. The reaction of lanthanide ions with r doxyl stearic acids and its utilization for the ESR study on the permeability of lipidbilayer of erythrocyte membrane to gadolinium ions. J Inorg Biochem, 1998, 69: 1 7

6 Cheng Y, Li Y, Li R C, et al. The uptake of cerium by erythrocytes and the changes of membrane permeability in CeCl ${ }_{3}$ feeding rats. Prog Nat Sci, 1999, 9(8): 610 616

7 程 驿, 刘峁子, 李 友, 等. 经消化道吸收的氯化钥诱导 Wistar 大鼠红细胞膜“畴” 结构的形成. 科学通报, 1999, 44(14): 1520 1523

8 Cheng Y, Yao H Y, Lin H K, et al. The events relating to lanthanide ions enhanced permeability of human erythrocyte membrane: binding, conformational change, phase transition, perforation and ion transport. Chem Biol Interact, 1999, 121: 267 289

9 Robert F. Drug delivery takes a deep breath. Science, 1997, 277: 1199 1200

10 Okumura K, Iwakawa S, Yoshida T, et al. Intratracheal delivery of insulin absorption from solution and aerosol by rat lung. Int J Pharm, 1992, 88: 63 73

11 Yamamoto A, Umemori S, Muranishi S, et al. Absorption enhancement of intrapulmonary administered insulin by various absorption enhancers and protease inhibitors in rats. J Pharm Pharmacol, 1994, 46: 14 18

12 Ando T, Maitani Y, Yamamoto T, et al. Nasal insulin delivery in rabbits using soybearr derived sterylglucoside and sterol mixtures as novel enhancers in suspension dosage forms. Biol Pharm Bull, 1998, 21: 862 865

13 Lee J, Pilch P F. The insulin receptor: structure, function, and signaling. Am J Physiol, 1994, 266: C319 C334

14 Wang L H, Wang S, Tang Y, et al. Effects of low concentration samarium chloride on serum hormone in rat. Chin J Rare Earths, 1996, 14: 186 188

15 Williams P F, Turtle J R. Terbium, a fluorescent probe for insulin receptor binding. Evidence for a conformational change in the receptor protein due to insulin binding. Diabetes, 1984, 33: 1106 1111 